\title{
Single-Cell Quantitative HER2 Measurement Identifies Heterogeneity and Distinct Subgroups within Traditionally Defined HER2-Positive Patients
}

\author{
Matthew D. Onsum, Elena Geretti, Violette Paragas, Arthur J. Kudla, Sharon P. Moulis, Lia Luus, Thomas J. Wickham, \\ Charlotte F. McDonagh, Gavin MacBeath, and Bart S. Hendriks
}

From Merrimack Pharmaceuticals, Cambridge, Massachusetts

Accepted for publication

July $16,2013$.

Address correspondence to Bart S. Hendriks, Ph.D., Merrimack Pharmaceuticals, 1 Kendall Sq, Suite B7201, Cambridge,

MA 02139. E-mail:

bhendriks@merrimackpharma. com.

\begin{abstract}
Human epidermal growth factor receptor 2 (HER2) is an important biomarker for breast and gastric cancer prognosis and patient treatment decisions. HER2 positivity, as defined by IHC or fluorescent in situ hybridization testing, remains an imprecise predictor of patient response to HER2-targeted therapies. Challenges to correct HER2 assessment and patient stratification include intratumoral heterogeneity, lack of quantitative and/or objective assays, and differences between measuring HER2 amplification at the protein versus gene level. We developed a novel immunofluorescence method for quantitation of HER2 protein expression at the single-cell level on FFPE patient samples. Our assay uses automated image analysis to identify and classify tumor versus non-tumor cells, as well as quantitate the HER2 staining for each tumor cell. The HER2 staining level is converted to HER2 protein expression using a standard cell pellet array stained in parallel with the tissue sample. This approach allows assessment of HER2 expression and heterogeneity within a tissue section at the single-cell level. By using this assay, we identified distinct subgroups of HER2 heterogeneity within traditional definitions of HER2 positivity in both breast and gastric cancers. Quantitative assessment of intratumoral HER2 heterogeneity may offer an opportunity to improve the identification of patients likely to respond to HER2-targeted therapies. The broad applicability of the assay was demonstrated by measuring HER2 expression profiles on multiple tumor types, and on normal and diseased heart tissues. (Am J Pathol 2013, 183: 1446-1460; http://dx.doi.org/10.1016/j.ajpath.2013.07.015)
\end{abstract}

Overexpression of human epidermal growth factor receptor 2 (HER2 or ErbB2) occurs in multiple human cancers and is associated with increased disease recurrence and poor prognosis. ${ }^{1,2}$ HER2-targeted therapeutics, such as trastuzumab and lapatinib, have demonstrated clinical benefit when combined with chemotherapies in HER2-positive breast and gastric cancer patients; however, HER2 testing continues to be controversial.

HER2 status, measured by immunohistochemistry (IHC) and/or fluorescent in situ hybridization (FISH), is an imprecise predictor of response to HER2-targeted therapy. For example, in HER2-positive metastatic breast cancer, response rates to trastuzumab-containing regimens vary from $36 \%$ to 79\%. ${ }^{4}$ Furthermore, some HER2-negative patients respond to trastuzumab. ${ }^{5}$ Beyond breast and gastric cancers, there are other solid tumors, such as bladder, ${ }^{6-8}$ endometrial, ${ }^{9-11}$ and lung, ${ }^{12-14}$ that have been shown to express HER2, and are not served by anti-HER2 therapy. These indications lack consensus for the best HER2 testing technique and for HER2 cutoffs to distinguish patient populations. ${ }^{15}$ Taken together, this suggests an opportunity for further improvement of HER2 testing methods. Challenges to correct HER2 assessment and patient stratification include intratumoral heterogeneity of HER2 expression, lack of objective HER2 quantification, and differences between measuring HER2

\footnotetext{
Supported by Merrimack Pharmaceuticals.

M.D.O., E.G., and V.P. contributed equally to this work.

Disclosures: All authors are employed by and hold stock options or bond holdings with Merrimack Pharmaceuticals.

Current address of M.D.O., Silver Creek Pharmaceuticals, Inc., San Francisco, CA.
} 
protein and DNA. We briefly review how existing IHC and FISH tests perform regarding these challenges.

Two IHC tests for assessing HER2 status have been approved by the Food and Drug Administration (FDA): HercepTest (Dako, Carpinteria, CA) and Pathway (Ventana Medical Systems, Tucson, AZ). Both of these tests use IHC staining of the HER2 protein and are then interpreted by a pathologist who scores the degree of staining as $0,1+, 2+$, or $3+$. Differences in sample handling, fixation, storage procedures, and staining have all been shown to interfere with the antigen retrieval, stability, and consequent reliability of IHC. ${ }^{16-20}$ In addition to issues of sample processing, the pathologist's interpretation can be subjective. Challenges in reproducibility across laboratories and pathologists, and even within pathologists themselves, further echo issues with the subjective nature of these tests. ${ }^{21-23}$ The greatest need for attention in HER2 assessment is regarding the scoring of samples with moderate levels of HER2. The variation in the percentage of samples scored as $2+$ across multiple studies is nearly five times that of $3+$ in both breast and gastric cancers $^{24}$ and, thus, has a large impact on which patients are considered eligible for anti-HER2 therapy.

Three FISH tests have been approved by the FDA for assaying HER2 gene amplification: PathVysion (Abbott, Abbott Park, IL), INFORM (Ventana Medical Systems), and PharmDx (Dako). HER2 gene amplification is assessed by counting HER2 foci within the nuclei of at least 20 cells in two distinct pathologist-assessed tumor areas. ${ }^{25}$ Centromere (CEP) 17 foci are also counted to report the ratio of HER2/CEP 17. CEP 17 serves as an internal control, something lacking in IHC tests. Recent reports, however, question the validity of using CEP 17 in conjunction with HER2. Although rare, polysomy of chromosome 17 has been shown to occur, ${ }^{26}$ and it is likely that increased signals of centromere 17 are the result of co-amplification with the HER2 gene. In these cases, patients would be incorrectly classified as nonamplified. ${ }^{27}$ According to the American Society of Clinical Oncology-College of American Pathologists (ASCO-CAP) guidelines, more than six gene copies of HER2 per nucleus or an HER2/CEP 17 ratio $>2.2$ should be scored as positive. ${ }^{28}$ Because of the counting of a few cells, FISH does not capture tumor heterogeneity. Furthermore, a recent analysis has suggested that looking at few cells can result in fluctuations of the HER2/CEP 17 ratio that could influence classification of a sample as HER2 amplified or not and the consequent therapeutic regimen for patient treatment. ${ }^{29}$ One advantage of FISH over IHC-based tests is that FISH results are less sensitive to sample handling and processing because HER2 DNA is more stable than HER2 protein. However, the effects of preanalytical variables on the performance of FISH have recently been at the center of controversy. Some reports showed no effects of ischemia time and time of fixation in $10 \%$ neutral-buffered formalin on FISH performance. ${ }^{20,30}$ In another study, Khoury et $\mathrm{al}^{31}$ demonstrated compromised FISH results by ischemia times longer than 1 hour. Similar to IHC, FISH interpretation requires a pathologist. Attempts have been made to automate FISH interpretation, ${ }^{32,33}$ but they have not yet been adopted into standard clinical practice. FISH has several drawbacks compared with IHC-based tests: It is more expensive, it is technically more cumbersome and time-consuming, and fewer laboratories have the ability to perform FISH. As a result, FISH is most commonly performed at centralized laboratories.

The extent to which tumor heterogeneity is prognostic or predictive of patient response to anti-HER2 therapy is not well understood, and no FDA-approved test is able to report a quantitative measure of HER2 heterogeneity. With current testing methods, it is not possible to systematically determine the optimal cutoffs for the percentage of cells expressing a certain level of HER2 expression for optimal patient responses. In breast cancer, ASCO-CAP guidelines recommend intense staining of $>30 \%$ of cells by IHC as a cutoff for positivity. By contrast, in gastric cancer, the recommended cutoff is $>10 \%$ of cells. ${ }^{28}$ Furthermore, HER2 expression in gastric tumors shows considerable intratumoral heterogeneity, accounting for a large portion of testing discordance. ${ }^{34,35}$

Previous work has described the development of automated quantitative analysis (AQUA) for measuring HER2 expression. ${ }^{36}$ AQUA uses a cytokeratin stain as a mask to identify tumor tissue in conjunction with anti-HER2 staining and detection via immunofluorescence. Relative quantitation of HER2 expression is performed using automated image analysis based on cell line standards in a tissueaveraged manner, and heterogeneity is reduced to a single variable using Simpson's biodiversity index. ${ }^{37,38}$ Herein, we extend that work by incorporating single cell-based image analysis that enables quantitation of HER2 protein expression and heterogeneity in terms of HER 2 number per cell at the single-cell level for use with clinical tumor samples.

\section{Materials and Methods}

\section{Materials}

RPMI 1640 medium, Leibovitz's L-15 medium, and McCoy's 5a medium modified were from Lonza (Walkersville, MD), fetal bovine serum was from Tissue Culture Biologicals (Seal Beach, CA), and a penicillin G/ streptomycin sulfate mixture was from GIBCO (Invitrogen, Grand Island, NY). Peroxidazed1, Background Sniper, and Da Vinci Green were from Biocare Medical (Concord, CA). Mouse anti-human pan cytokeratin and Envision anti-rabbit horseradish peroxidase were from Dako Cytomation (Carpinteria). Rabbit anti-human HER2 (clone SP3), Tris-EDTA buffer $(\times 100$ magnification), and Tris-buffered saline with Tween 20 buffer $(\times 20$ magnification) were from Fisher Scientific (Pittsburgh, PA). TSA Cyanine 5 Tyramide Reagent was purchased from PerkinElmer Life Sciences (Waltham, MA). Goat antimouse Alexa 555 and ProLong Gold with DAPI were from Invitrogen (Carlsbad, CA). 
Tissue Culture

DU145, MDA-MB-175-VII, MDA-MB-453, ACHN, and SKOV3 cells were obtained from ATCC (Manassas, VA) and grown under recommended conditions. IGROV1 was from NCI-DTP (Bethesda, MD). BT474-M3 cells are an HER2overexpressing cell line gift from Hermes Biosciences (San Francisco, CA). MCF-7 clone 18 cells were a gift from Dr. Christopher Benz (Buck Institute, Novato, CA).

\section{HER2 Quantification in Cell Lines Using qFACS}

Cells were trypsinized, washed, and stained using fluorescently labeled trastuzumab. Trastuzumab was labeled as previously described. ${ }^{39}$ HER2 receptor numbers were determined by assessing the antibody-binding capacity of the fluorescently labeled HER 2 antibody via quantitative fluorescence-activated cell sorting. Antibody-binding capacity was determined using Simply Cellular Quantum Beads (Bangs Labs, Fishers, IN), per the manufacturer's instructions.

\section{Cell Pellet Array}

For each cell line, $2.5 \times 10^{8}$ cells at $80 \%$ confluence were rinsed with phosphate buffered saline (PBS) and covered with $10 \%$ neutral-buffered formalin at room temperature for 10 minutes with gentle agitation. Cells were collected by scraping, pelleted at $208 \times g$ for 10 minutes at $4^{\circ} \mathrm{C}$, and resuspended in $70 \%$ ethanol. Cells were pelleted and transferred into an Eppendorf tube prepared with a bed of paraffin. Cells were packed by centrifuging at $11,758 \times g$ for 5 minutes at room temperature, followed by aspiration of the ethanol. Cell pellet molds were prepared by placing Eppendorf tubes in an embedding mold and surrounding with molten $\left(55^{\circ} \mathrm{C}\right) 1 \%$ low-melt agarose in Tris-buffered saline and allowed to set. Cell pellets were placed in the center of the agarose mold and sealed with agarose. Agaroseembedded cell pellets were immersed in $70 \%$ ethanol at $4^{\circ} \mathrm{C}$ until being embedded in paraffin and divided into sections (Mass Histology Service, Inc., Worcester, MA).

\section{Patient Samples}

Sections $(5 \mu \mathrm{m}$ thick) of a breast disease spectrum TMA were obtained from Folio Biosciences (Powell, $\mathrm{OH}$ ) (catalog number ARY-HH0137). Duplicate 1-mm tissue cores from 48 patients were represented on the TMA. Individual sections (5 $\mu \mathrm{m}$ thick) of 179 gastric and 57 gastroesophageal junction (GEJ) tumors were acquired from Cureline (South San Francisco, CA) and Folio Biosciences. Sections (5 $\mu \mathrm{m}$ thick) of a bladder carcinoma progression TMA (catalog number ARY-HH0150) and of an ovary carcinoma high-density TMA (catalog number ARY-HH0180) were from Folio Biosciences. Sections (5 $\mu \mathrm{m}$ thick) of a heart disease and normal TMA (catalog number BC30013) were from US Biomax (Rockville, MD).
Immunofluorescence Staining and Image Acquisition

The cell pellet array and a breast cancer TMA were stained with an anti-human pan cytokeratin antibody and an antihuman HER2 antibody, as follows. Slides were baked for 30 minutes at $65^{\circ} \mathrm{C}$ and deparaffinized by immersion in xylene ( 2 times for 30 minutes each), 100\% ethanol (2 times for 2 minutes each), and $80 \%$ ethanol ( 2 times for 2 minutes each), followed by water. Antigen retrieval was accomplished by heating the slides in Tris-EDTA buffer, $\mathrm{pH} 9$, for 25 minutes at $95^{\circ} \mathrm{C}$ in a pretreatment module (Thermo Scientific, Waltham, MA). After antigen retrieval, slides were stained on a Lab Vision Autostainer 360 (Thermo Scientific). Briefly, endogenous peroxidase activity was blocked with Peroxidazed 1 (10 minutes at room temperature), followed by a washing step with Tris-buffered saline with Tween 20 and a protein blocking step with Background Sniper (10 minutes at room temperature). Next, slides were incubated with the mouse anti-human pan cytokeratin and rabbit anti-human HER2 antibodies diluted in Da Vinci Green for 1 hour at room temperature. After washing, slides were incubated with a goat anti-mouse Alexa 555 antibody diluted in Envision anti-rabbit horseradish peroxidase for 30 minutes at room temperature. After washing, incubation with TSA Cyanine 5 Tyramide Reagent for 10 minutes at room temperature followed. Slides were washed and mounted with ProLong Gold mounting media with DAPI. For the quantification of HER2 on human heart tissue specimens, a heart TMA containing both normal and diseased tissues and a cell pellet array were stained as previously described, with the omission of the cytokeratin antibody. Cell pellet arrays and TMAs were scanned on an Aperio ScanScope FL (Aperio, Vista, CA) at $\times 20$ magnification with a 0.75 Plan Apo objective. Images were acquired using Semrock filters DAPI-1160B-AMFZERO, SpOr-B-AMF-ZERO, and Cy5-4040C-AMF-ZERO specific for the DAPI, Alexa 555, and Cy5 signals, respectively (Semrock Inc., Rochester, NY). The Alexa 555 signal of cytokeratin and the Cy5 signal of HER 2 are pseudocolored in green and red, respectively, in the figures presented.

\section{Automated Image Analysis}

Automated image analysis was performed using custom rule sets written in Definiens Developer XD (Definiens, Munich, Germany). Briefly, nuclei were segmented in the DAPI layer. Subsequently, cells were identified by growing the nuclei until reaching the edge of the cytokeratin signal. The cytokeratin signal was used to distinguish between tumor cells (cytokeratin positive) and non-tumor cells/stroma (cytokeratin negative). The intensity of the HER2 membrane staining was quantified on a single-cell basis as follows: (mean of the inner border of the HER2 layer) + (mean of the outer border of the HER2 layer). For the quantification of HER2 on heart tissue samples, and relative standard pellet array, a modification of the previously described analysis was used in that, after nuclei detection, cells were outgrown until reaching the 
HER2 membrane staining. The intensity of the HER2 staining was quantified and used to classify cells into HER2positive and HER2-negative cells. In the case of the cell pellet array, the values of the mean HER2 membrane intensities of the cores of the different cell lines were exported and plotted against the corresponding log (HER2 receptor numbers) determined by quantitative fluorescence-activated cell sorter (qFACS) to generate a standard curve. In the case of the TMAs, the HER2 membrane staining intensity values of each single tumor cell of the core was exported and further analyzed based on the generated standard. Rule sets are available on request.

\section{HER2 IHC Testing}

Patient tumor samples were tested with the HercepTest (Dako), according to the manufacturer's directions, performed by Folio Biosciences. The TMA was scored using the ASCO-CAP guidelines for HercepTest interpretation. ${ }^{28}$

\section{HER2 FISH Testing}

FISH analysis was performed at the Dana-Farber/Harvard Cancer Center Cytogenetics Core Facility (Brigham \& Women's Hospital, Boston, MA). A breast cancer TMA was hybridized with a two-color commercial FISH probe (PathVysion HER2 DNA Probe Kit; Abbott Molecular, Des Plaines, IL) containing the HER2/neu region (SpectrumOrange), and a chromosome 17 enumeration probe, CEP 17 (SpectrumGreen). Control slides (Abbott Molecular) were run concurrently with the breast cancer TMA. The assay was performed according to the manufacturer's instructions. Stained slides were imaged on an Olympus BX51 microscope, using a chargecoupled device camera (ER3339) and CytoVision 3.6 Build 16 imaging software, both supplied by Applied Imaging Corp (Grand Rapids, MI). The TMA cores were initially imaged in the DAPI channel at low power through a $10 \times$ objective, to identify the tumor areas. Subsequently, actual scoring was accomplished by imaging through a $100 \times$ oil objective. According to the Abbott guidelines and consistent with the ASCO-CAP guidelines, ${ }^{28}$ analysis of the TMA cores was performed by scoring a minimum of 20 identifiable tumor cell nuclei, or if no obvious tumor was identified after scanning the entire core, 20 ductal cells were scored. If neither tumor nor ductal cells could be identified, the core was considered nonanalyzable. Cores with an HER2/CEP 17 ratio of $<1.8$ were considered nonamplified, and those with an HER2/CEP 17 ratio of $>2.2$ were considered amplified. Cores with an HER2/CEP 17 ratio between 1.8 and 2.2 were considered borderline amplified, and additional cells were scored. Cores with no visible signal in one or both hybridization colors were considered not analyzable.

\section{Data Analysis}

Output from the automated image analysis of patient samples and cell pellet arrays (standards) was analyzed using
MATLAB version R2011a (The MathWorks, Natick, MA). Calibration curves of mean fluorescence intensity (MFI) to log-transformed HER2 receptor number were generated using linear regression on the quantified images from the cell pellet array. For each TMA, a cell pellet array was stained in parallel, and the resulting calibration curve was unique to that TMA. HER2 membrane staining intensities on a per-cell basis from the TMAs were interpolated based on the calibration curve from the corresponding cell pellet array. The distribution of HER2 receptor numbers for the tumor cells in a TMA core was represented with the complementary cumulative distribution (or tail distribution). This representation facilitated the identification of the percentage of cells within a core that exceeded a given HER2 receptor value. Subgroups of HER2 expression patterns were identified by clustering the probability density estimates of HER2 expression of the tumor samples. Probability density estimates for each tumor sample were obtained by normal kernel density estimation, and the HER 2 probability density estimates were clustered with the K-means algorithm. The number of seed clusters for the K-means algorithm was determined empirically: several initial seed values were attempted, and the resulting clusters were assessed by the sum of the within-cluster point-to-centroid distances.

\section{Generation of Tumor Xenografts and Tissue Collection}

The 7-week-old female NCR/nu nude mice were purchased from Taconic (Germantown, IN). In accordance with the Public Health Service Policy and the Guide for the Care and Use of Laboratory Animals, all resident colony animals received acceptable standards in their care, use, and treatment. The care and treatment of experimental animals was in accordance with Institutional Animal Care and Use Committee guidelines.

BT474-M3 $\left(15 \times 10^{6}\right)$ and SUM190 $\left(10 \times 10^{6}\right)$ tumor cells were inoculated into the mammary fat pad of nu/nu mice. MDA-MB-453 cells $\left(10 \times 10^{6}\right)$ were inoculated s.c. into the right flank of nu/nu mice. When BT474-M3 $(n=5)$ and MDA-MB-453 $(n=3)$ tumors reached a volume of approximately $300 \mathrm{~mm}^{3}$, mice were sacrificed in a $\mathrm{CO}_{2}$ chamber and tumors were collected and fixed immediately in $10 \%$ formalin for 24 hours and subsequently processed to generate formalin-fixed, paraffin-embedded (FFPE) tumor sections. In the case of the SUM190 tumors, to generate enough material, tumors were allowed to grow until approximately $2000 \mathrm{~mm}^{3}$. At that point, mice were sacrificed in a $\mathrm{CO}_{2}$ chamber, tumors $(n=2)$ were collected, and each of them was divided into pieces that were kept either at $4^{\circ} \mathrm{C}$ (samples 1 to 13) or at room temperature (samples 14 to 26) during ischemia time. The details of the different ischemia times and fixation times are described in Supplemental Table S1. After fixation, tumor fragments were processed for FFPE sections. Three identical sets of slides were generated. Two sets were paraffin dipped and stored either in the desiccator or on the bench top for 3 weeks. The third set of slides was not 


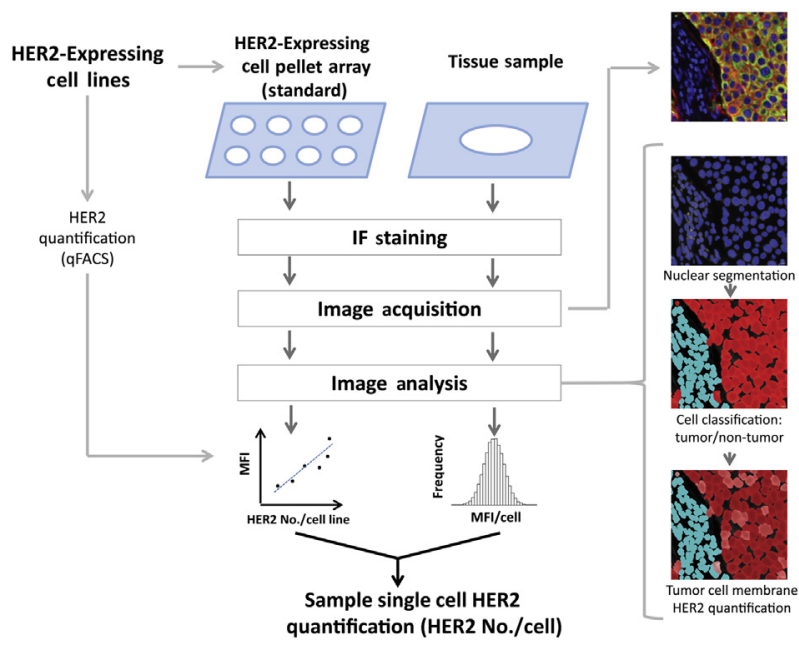

Figure 1 Schematic overview of the quantitative immunofluorescence (IF) assay. Cell lines with a range of HER2 expression, as quantified by qFACS, are used to generate a cell pellet array standard. The standard is stained in parallel with a tissue of unknown HER2 expression. Images of the standard and of the tissue are acquired and analyzed by automated image analysis tools. Analysis allows for the generation of a standard curve that can be used to interpolate HER2 numbers on a cell-by-cell basis on the tissue of unknown HER2 expression.

paraffin dipped and was stored on the bench top for 3 weeks until staining and subsequent analysis.

\section{Results}

\section{Assay Development}

Because anti-HER2 therapeutics act at the protein level, we sought to design an assay for protein detection, and couple it with a quantitative and objective analysis method. A highlevel overview of the assay is shown in Figure 1 and is described in detail later.

\section{Cell Pellet Microarray Generation}

Absolute quantitation of HER2 from a tissue section required the generation of a standard curve that related tissue staining to HER2 receptor levels. The standard curve was generated by measuring HER2 expression levels in a panel of cell lines, and then by generating an array from pellets of these cells to be stained in parallel with the tissue sample of interest. Eight cell lines (ACHN, DU145, IGROV1, MDA-MB-175-VII, MDA-MB-453, MCF7-clone 18, SKOV3, and BT474-M3) were selected to span a wide range of HER2 expression, as measured by qFACS (Table 1). All cell lines included in the cell pellet array had a single HER 2 population, as evaluated by FACS. Cores from each of the cell line-derived cell pellets were placed on the array in quadruplicate. The completed cell pellet microarray was divided into sections and stained in parallel with tissue samples of unknown HER2 expression levels.

\section{Staining and Image Analysis}

The cell pellet array standard and TMAs were stained in parallel to ensure consistency of staining. The staining was performed as described in Materials and Methods with an anti-human pan cytokeratin antibody to distinguish tumor from nontumor cells and an anti-human HER2 antibody to identify HER2, and counterstained with DAPI to identify cell nuclei. Images of entire sections of the cell pellet array and TMAs were digitally acquired for subsequent analysis. Representative images of the cell pellet array cores of the different cell lines are shown in Figure 2A.

Following image acquisition, automated image analysis was used to quantify the HER2 staining of each cell within the cell pellet array and the TMA. The same image analysis algorithm was applied to both the cell pellet standards and the TMA. The analysis consisted of the following: i) cell segmentation using the nuclear stain, ii) identification of tumor and non-tumor cells based on the cytokeratin stain, and iii) quantification of HER2 staining along the membrane on a cell-by-cell basis for all of the identified tumor cells. The algorithm was designed to obviate the need for user input and maximize objectivity. Segmentation and classification of the cells within the cell pellet microarray are shown in Figure 2A. Tumor cells were classified into HER2 low ( $<$ approximately 150,000 HER2 per cell; pink), HER2 medium ( $>$ approximately 150,000 and $<$ approximately 1,000,000 HER2 per cell; light red), and HER2 high ( $>$ approximately 1,000,000 HER2 per cell; red). HER2negative cells are shown in gray. The distribution of the HER2 membrane staining intensity per cell for all of the cell lines of the standard array is shown in Figure 2B and indicates single populations of HER2 expression, consistent with observations by qFACS (data not shown).

\section{Assay Qualification}

\section{Standard Curve}

Image analysis of the cell pellet array enables calculation of the average HER2 MFIs for each cell line. These values were combined with qFACS measurements to generate a standard curve of MFI versus HER2 expression in terms of receptors per cell (Figure 2C). Based on goodness of fit and an analysis of residuals, it was determined that a log-linear calibration model gave the most robust and parsimonious fit to the data (Figure 2D), yielding a measurement accuracy of $\pm 10 \%$ to $15 \%$ receptors per cell. Based on the standard curve, the lower and upper limits of quantitation were

Table 1 Cell Lines Selected for the Standard Cell Pellet Array and Corresponding HER2 Receptor Numbers as Determined by qFACS

\begin{tabular}{lc}
\hline Cell line & Mean HER2 (No./cell) \\
\hline ACHN & 45,000 \\
DU145 & 69,000 \\
IGR0V1 & 158,000 \\
MDA-MB-175-VII & 202,000 \\
MDA-MB-453 & 393,000 \\
MCF7 clone18 & $1,030,000$ \\
SKOV3 & $1,380,000$ \\
BT474-M3 & $1,940,000$ \\
\hline
\end{tabular}



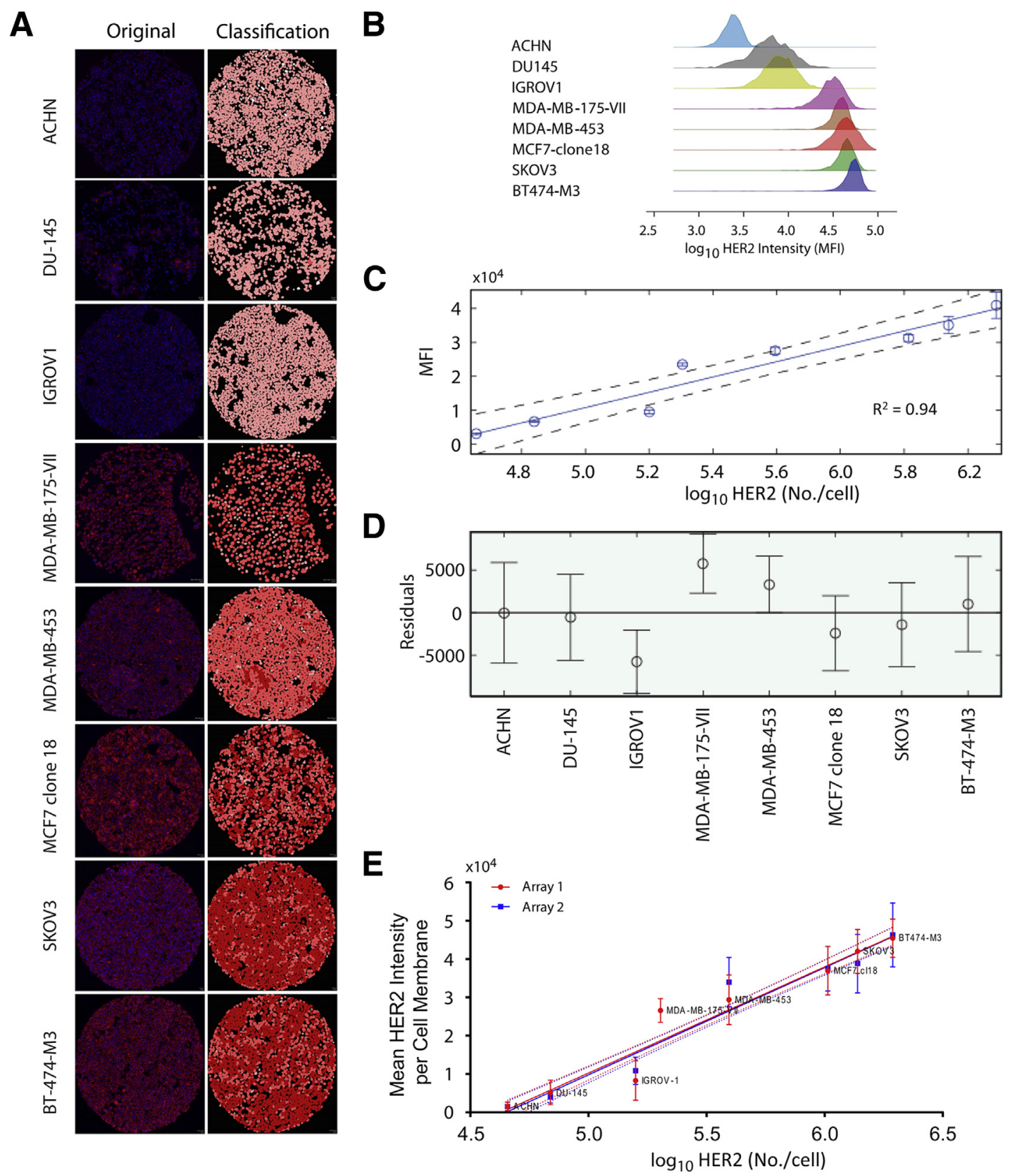

Figure 2 A: The cell pellet array was stained with an anti-HER2 and anti-pan cytokeratin antibody and counterstained with DAPI. The slide was scanned with an Aperio ScanScope FL, and resulting images were analyzed with Definiens Developer XD. The original and classification views of representative cores of the different cell lines of the cell pellet array are shown. The cytokeratin layer was omitted from the original views, and only the HER2 (red) and DAPI (blue) layers are shown for clarity of visualization. The classification views include HER2 low-expressing cells ( $<$ approximately 150,000 HER2 per cell) (pink), medium HER2-expressing cells (> approximately 150,000 and < approximately 1,000,000 HER2 per cell) (light red), high HER2 (> approximately 1,000,000 HER2 per cell) (red), and HER2-negative cells (gray). B: Representation of the single-cell distribution of the $\log _{10}$ (mean HER2 membrane intensity per cell) for the different cell lines of the standard. C: The mean HER2 membrane intensity per core of each cell line is plotted against the corresponding log ${ }_{10}$ HER2 receptor numbers, quantified by qFACS, to generate a standard curve. Dashed lines indicate $95 \%$ CI. The curve was analyzed with a linear regression fit $\left(R^{2}=\right.$ 0.94). D: The regression residuals (with $95 \%$ CIs) are plotted for each cell line. E: Slides from two cell pellet arrays (1, red; and 2, blue), generated by two different batches of cell line collections, were stained on multiple days by different investigators ( $n=10$ repeats per array). The averages of the 10 repeats are shown for both arrays 1 and 2 . The two curves were analyzed with a linear regression fit $\left(R^{2}=0.875\right.$ for array 1 , and $R^{2}=0.874$ for array 2$)$. The $95 \%$ CI is represented by the dashed lines (red for array 1 and blue for array 2).

approximately 45,000 and 1,900,000 receptors per cell, respectively, varying slightly across staining runs.

Furthermore, we evaluated the effects of different batches of cell line collection on the resulting standard curve. The cell lines selected for the cell pellet array were collected at two different times by different investigators (L.L., V.P., A.J.K., and E.G.), and were used to generate two distinct cell pellet arrays (array 1 and array 2). Slides from the two different batches were stained on multiple days by different investigators (V.P. and E.G.), and were analyzed as 

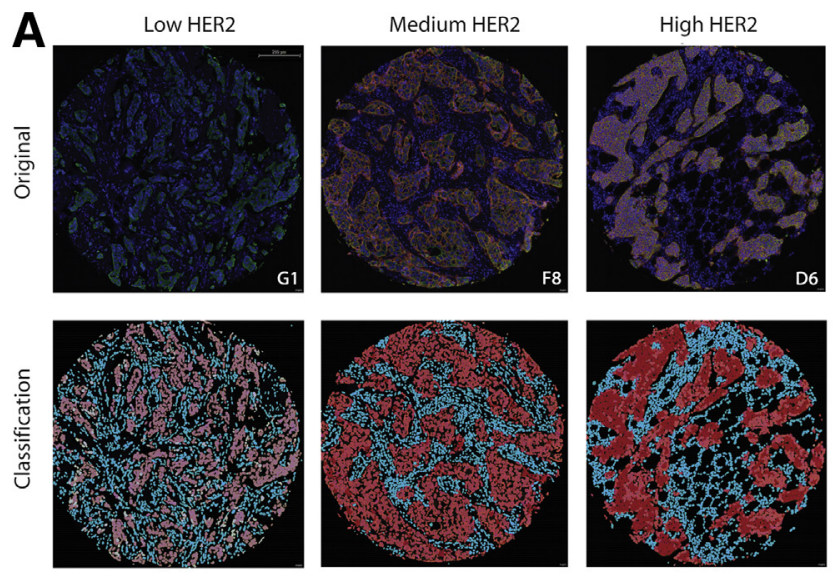

HER2 high cell

HER2 medium cell

HER2 low cell

Stroma cell
Figure 3 A: A breast disease TMA was stained with HER2 (red), cytokeratin (green), and DAPI (blue). Representative TMA cores at low $\left(\mathrm{G}_{1}\right)$, medium $\left(\mathrm{F}_{8}\right)$, and high $\left(D_{6}\right)$ HER2 expression are shown with the corresponding cell segmentation and classification. Pink indicates low HER2 expression ( $<$ approximately 150,000 HER2 per cell), light red indicates medium HER2 expression (> approximately 150,000 and < approximately $1,000,000$ HER2 per cell), red indicates high HER2 expression (> approximately 1,000,000 HER2 per cell), and cyan represents non-tumor cells/stroma. The mean HER2 receptor numbers per cell, interpolated based on the standard cell pellet array stained in parallel with the TMA, are shown in B. C: The distribution of HER2 expression among the different populations (HER2 high, red; HER2 medium, light red; HER2 low, pink; and HER2 negative, white) is shown for all of the TMA cores.

B
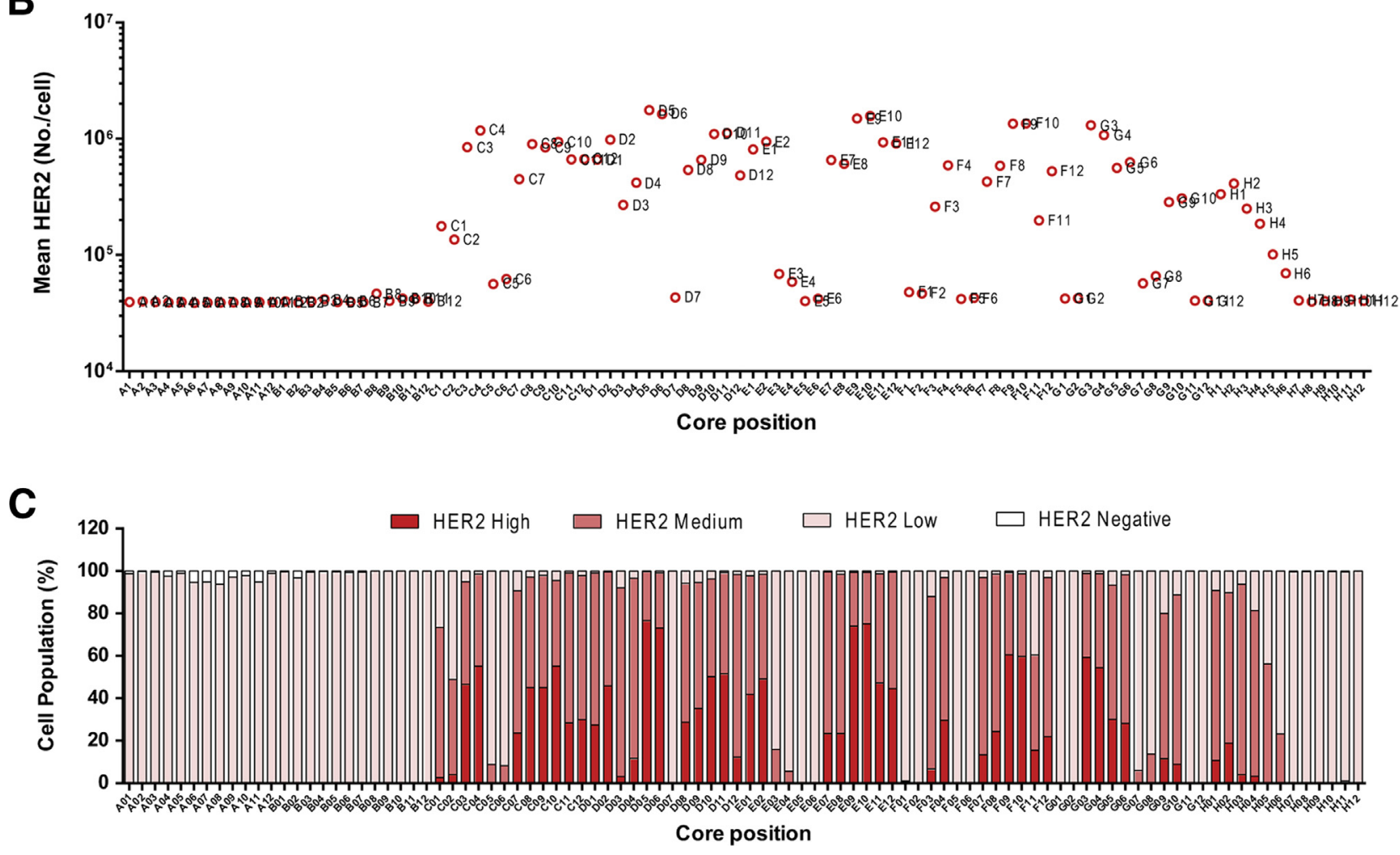

previously described. An average of the analysis of 10 slides from array 1 and 10 slides from array 2 is shown in Figure 2E. No significant differences were observed in the resulting standard curves of the same array (1 or 2) run on multiple days. The log-linear fits of the two data sets were essentially overlaying. Both the slopes $(27,700 \pm 1200$ for array 1 and 28,000 \pm 1300 for array 2) and the $y$-intercepts $(-128,000 \pm 6700$ for array 1 and $-130,000 \pm 7200$ for array 2) were not significantly different, indicating there is minimal batch-to-batch variability in this assay.

To evaluate potential differences in antigen recognition in the cell pellet array (used as a standard) versus tissue, we stained the cell pellet array in parallel with slides from BT474-M3 xenograft tumors $(n=5)$. The BT474-M3 xenograft tumors were generated by growing in vivo an aliquot of BT474-M3 cells originating from the same original batch of cells used to generate the BT474-M3 cores in the standard cell pellet array. After staining and image analysis, the mean HER2 receptor number per cell was interpolated for the individual cores of the BT474-M3 cells within the cell array and for the individual BT474-M3 tumor xenografts, and the results are shown in Supplemental Figure S1A. There was no significant difference between the mean HER2 receptor number per cell of the BT474-M3 cells within the array $(2,018,000 \pm 260,000)$ and within the BT474-M3 tumor xenografts $(2,050,000 \pm 698,000)$.

\section{Sample Analysis}

A breast disease spectrum TMA with 48 patient samples represented in duplicate (total of 96 cores) that included 

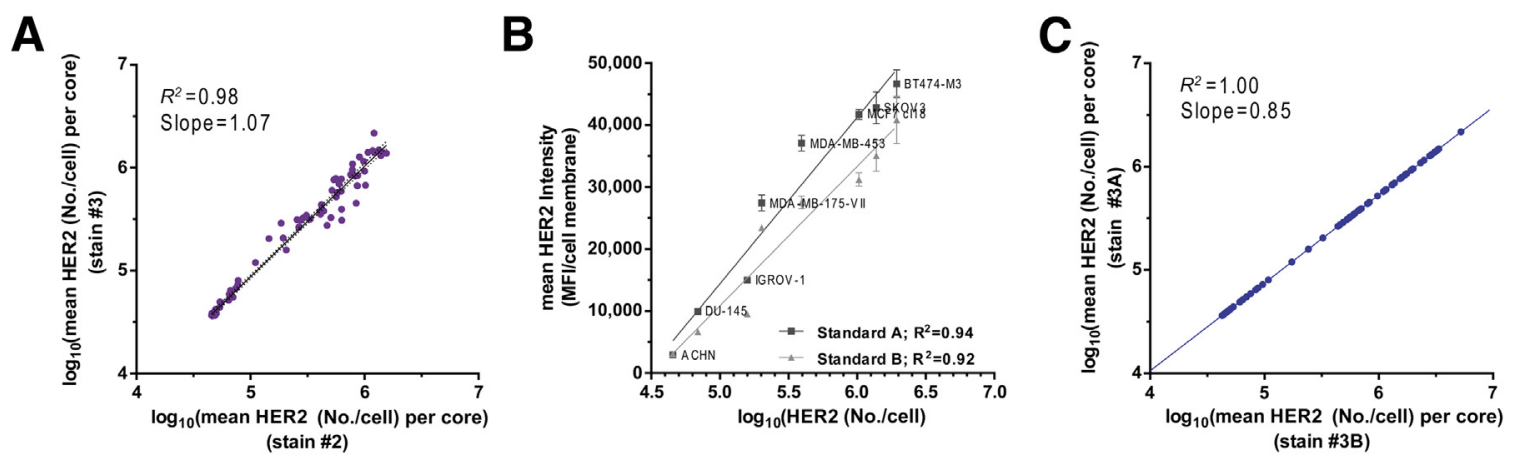

Figure 4 A: Two consequent sections of the breast disease TMA were stained in parallel with a standard cell pellet array on two separate days. The mean HER2 per cell on each individual core for the two different TMA sections was interpolated from the corresponding standard and plotted against one another. The data were fit with a linear regression giving an $R^{2}$ of 0.98 and a slope $=1.07$. B: Two standard pellet arrays (standard A and standard B) were stained on the same day. The mean HER2 intensities per cell membrane for the different cell lines of the standard were plotted against the correspondent $\log _{10}$ HER2 receptor number determined by qFACS. The standards were fitted in GraphPad Prism (La Jolla, CA) with a linear regression ( $R^{2}$ of 0.94 and 0.92 for standards A and B, respectively). C: A breast disease TMA was stained in parallel with the two previously described standard cell pellet arrays. The mean HER2 receptor numbers for the TMA cores were interpolated from either standard and were plotted against each other. The data were fit with a linear regression $\left(R^{2}\right.$ of 1.00 , slope $\left.=0.85\right)$.

different stages of breast cancer and breast cancer types, as well as normal breast tissue (as control), was used for subsequent analysis. The TMA was stained for HER2 and cytokeratin, and counterstained with DAPI. Staining of the breast disease TMA was run in parallel with the standard cell pellet array. Three tumor cores are shown with the corresponding cell segmentation by automated image analysis (Figure 3A). By using the standard curve, mean HER2 expression numbers per cell were calculated for each individual core (Figure 3B). The percentages of the different tumor cell populations for each of the breast disease TMA cores are shown in Figure 3C.

\section{Assay Reproducibility}

We assessed the technical reproducibility of our assay by comparing the mean HER2 per cell values determined from

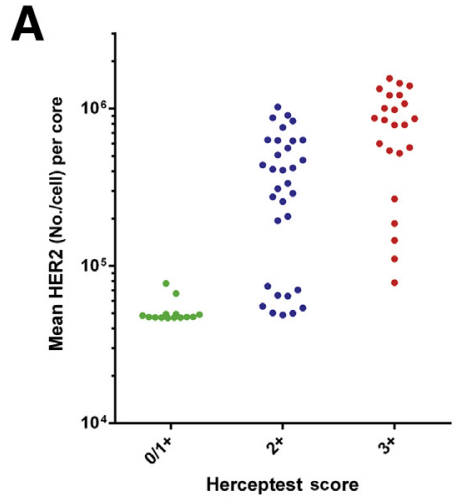

D

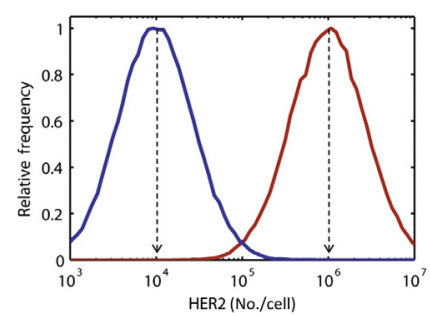

B

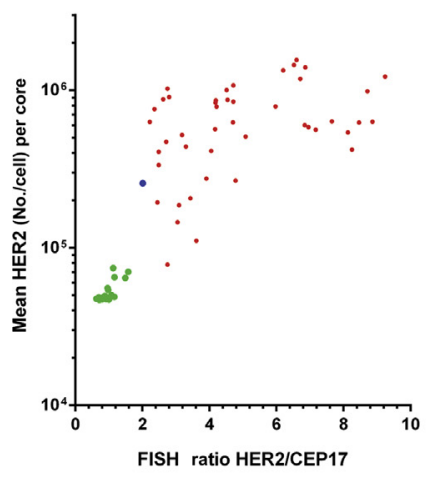

E

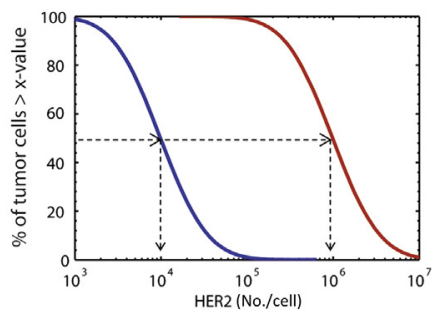

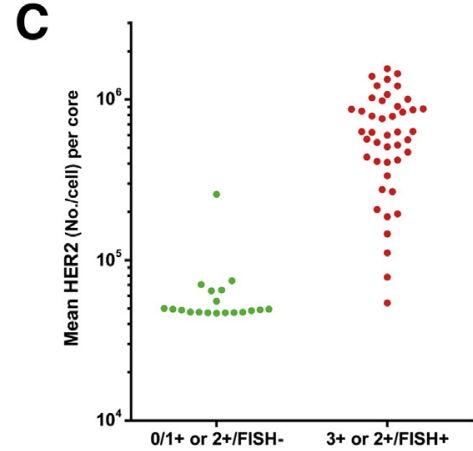

$\mathbf{F}$

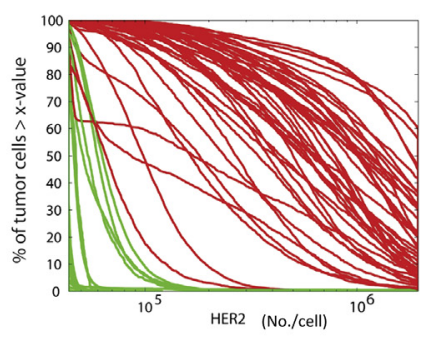

Figure 5 A: The mean HER2 receptor numbers per core are plotted against the HercepTest scores determined by a pathologist as $0 / 1+(\mathrm{green}), 2+$ (blue), and $3+($ red). B: The mean HER2 receptor numbers per core are plotted against the HER2/CEP 17 ratios obtained from FISH staining of a nearby region tissue slide, and scored by a pathologist as FISH positive (red), FISH negative (green), or borderline (blue). C: The mean HER2 receptor numbers per core are plotted against the HercepTest and FISH scores determined by a pathologist, based on which cores are classified into HER2 negative $(0 / 1+/ 2+$ and FISH negative; green) and HER2 positive (2+ and FISH positive and 3+; red). D: An example distribution of HER2 expression (HER2 numbers per cell) is shown. E: The data in D were replotted using an inverse cumulative distribution. At the $Y$ value of 50,50\% of the cells of the core represented in blue express $>10,000 \mathrm{HER} 2$ per cell, and $50 \%$ of the cells of the core represented in red express $>1,000,000$ HER2 per cell. F: The HER2 expression on all of the tumor cells of each individual core of the breast disease TMA is plotted as an inverse cumulative distribution and color coded as in $\mathbf{C}$. 

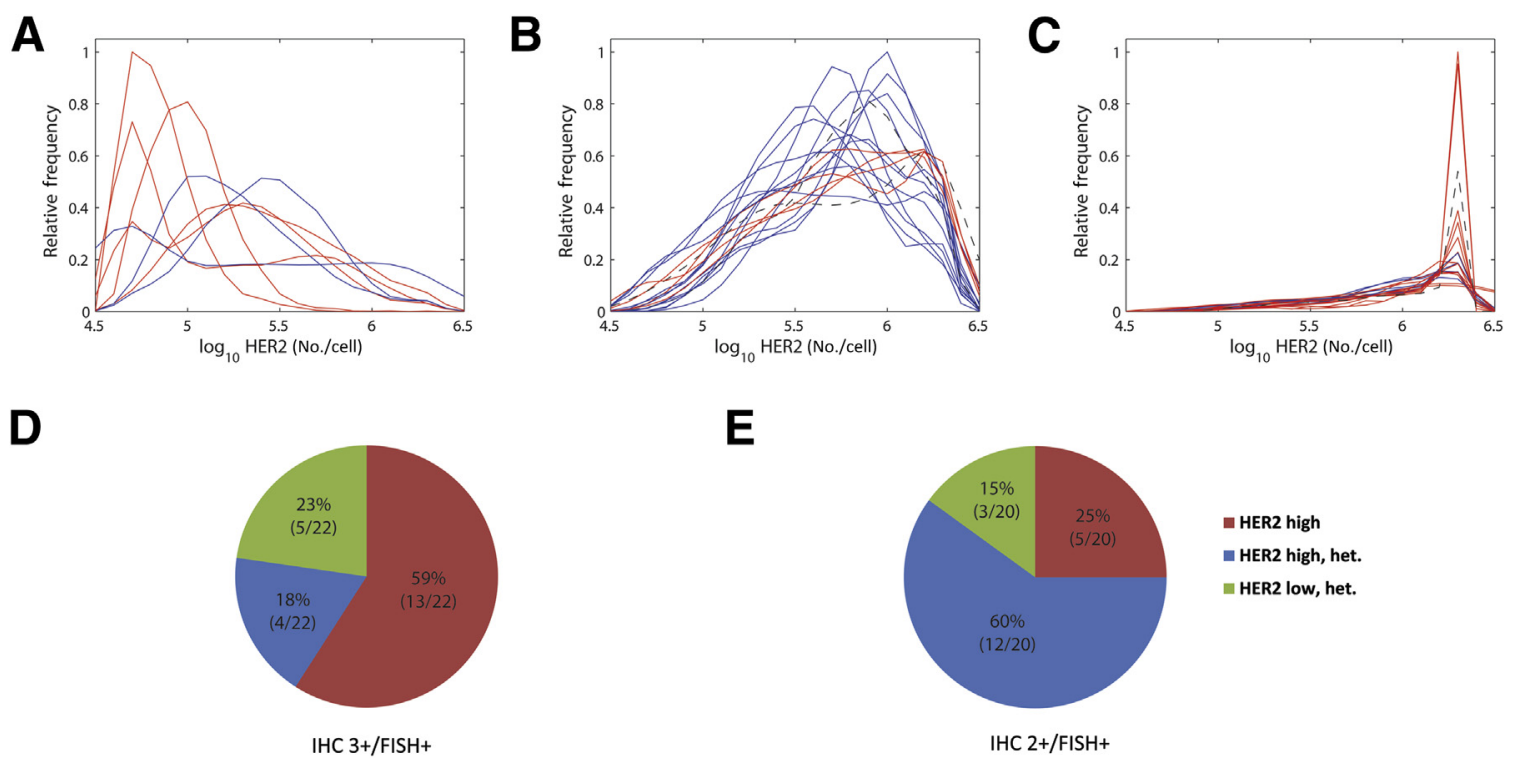

Figure 6 HER2-positive TMA cores clustered into three distinct patterns of HER2 protein expression. The histograms of HER2 expression for the subgroups (A), HER2 low and heterogeneous (het.); these samples are HER2 positive in a clinical sense but have an overall lower expression and show heterogeneity with a dominant peak of lower-expressing cells. B: HER2 high and heterogeneous; these samples are dominated by high HER2-expressing cells, but still have a significant amount of heterogeneity. C: HER2 unambiguously high; these samples show high and uniform HER2 expression with little heterogeneity. The line colors in A-C correspond to the IHC score for each sample (red, 3+; dark blue, 2+). Frequency represents probability density-the $y$ axis is normalized such that the area under the density curve is unity. The pie charts in $\mathbf{D}$ and $\mathbf{E}$ show the relative abundance of these subgroups in the IHC $3+$, FISH+, and IHC $2+$, $\mathrm{FISH}+$ categories, respectively (unambiguously high, dark red; high and heterogeneous, blue; low and heterogeneous, light green).

near-identical sections of the breast disease TMA previously described. Consequent sections of the TMA were stained in parallel with a control array standard on different days, and the HER2 per cell levels for the individual cores of the array were determined. Mean HER2 per cell values for each of the 96 cores on the breast disease TMA from the two sections are shown in Figure 4A to aid in visual comparison. The overall concordance was excellent, with $R^{2}=0.98$ and slope $=1.07$.

The effect of the standard curve on the calculated HER2 per cell values was investigated by staining two control arrays in parallel with a single breast disease TMA. Shown in Figure 4B are the two standard curves obtained from the staining of two control arrays (standard A and standard B). After simultaneous interpolation of the mean HER2 per cell for each of the 96 individual cores from either standard A or standard B, a correlation plot was generated and is shown in Figure 4C. A high overall concordance between the two interpolations was observed $\left(R^{2}=1.00\right.$, slope $\left.=0.85\right)$. From these data, we can estimate the uncertainty in mean HER2 per cell derived from variability in cell standards to be approximately $15 \%$, a highly accurate measurement considering that HER2 protein expression ranges across three to four logs.

\section{Single-Cell Analysis of Patient Samples}

\section{Comparison with Traditional HER2 Classification}

The breast disease spectrum TMA used for Figures 3 and 4 contains samples from normal breast and a variety of stages of breast cancer. Consequently, it is not expected to capture the typical distribution of HER2 positivity, in terms of either HercepTest or FISH scores, as reported by broad-based surveys of HER2 expression. However, it is well suited for comparison of HER2 expression measured by our assay with clinically used tests. HercepTest and FISH were performed on serial slides of the breast disease spectrum TMA. The mean HER2 receptor numbers per core are plotted against the HercepTest scores $(3+$, red; $2+$, dark blue; $1+/ 0$, green) in Figure 5A. An overall correlation between high HER2 receptor numbers and high $(3+)$ scores was observed. However, cores scoring for a particular value by HercepTest span a wide variety of interpolated mean HER2 receptor numbers. The interpolated mean HER2 receptor numbers per core are plotted against the corresponding ratio of HER2/CEP 17 for each individual core in Figure 5B (FISH positive, red; borderline, blue; FISH negative, green). Cores with a high level of amplification were also characterized by a high mean HER2 receptor number, as determined by our assay, but this correlation did not hold for all of the FISH amplified cores. After the clinical HER2 classification scheme, individual samples within the TMA were separated into two groups: those that would not be eligible for anti-HER 2 therapy $(0 / 1+l$ $2+$ and FISH negative; HER 2 negative) and those that would be eligible for anti-HER 2 therapy ( $2+$ and FISH positive and $3+$; HER2 positive). The mean HER2 receptor numbers per core are plotted against these traditional definitions of HER2 in Figure 5C. From the analysis of receptor numbers, there is a clear distinction between the two groups, based on the combination of HercepTest and FISH testing. 
A

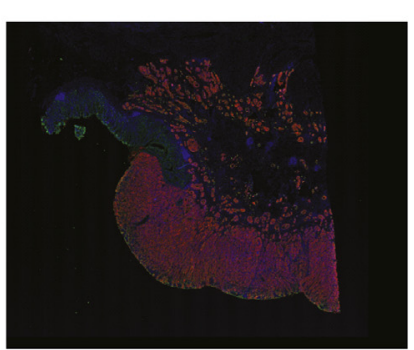

C

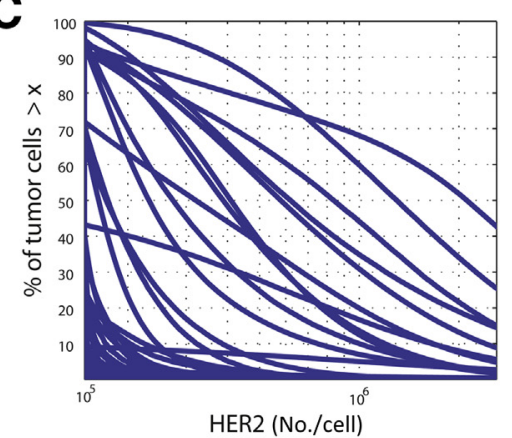

E

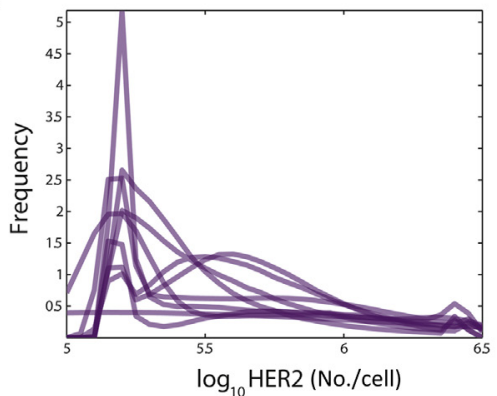

B

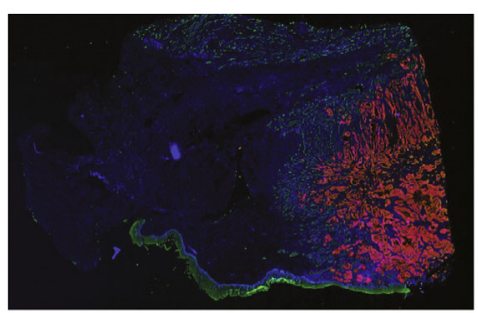

D

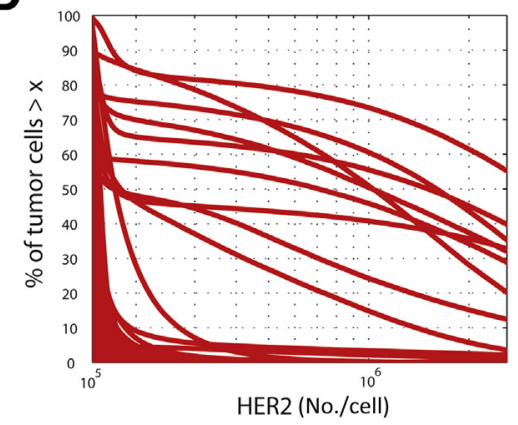

$\mathbf{F}$

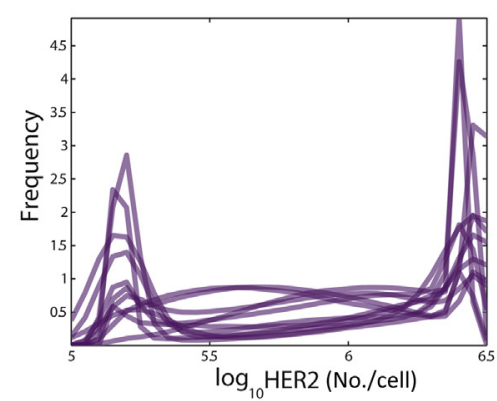

Figure 7 Assessment of HER2 expression and heterogeneity in gastric and GEJ whole tumor sections. Representative gastric (A) and GEJ (B) tumor sections stained with HER2 (red), cytokeratin (green), and DAPI (blue). The HER2 expression on all tumor cells in each individual tumor section for gastric (C) and GEJ (D) plotted as inverse cumulative distributions. Gastric and GEJ HER2 expression profiles clustered into three distinct subgroups: unambiguously low (data not shown), samples that show low and uniform expression of HER2; low and heterogeneous, samples characterized by having low but measurable numbers of high HER2-expressing cells (E); and high and heterogeneous, samples with more high HER2-expressing cells, but still far from uniformly high expressing (F).

\section{Data Visualization}

To realize the full potential of our assay, the method used to visualize single-cell HER2 expression is critical. To demonstrate this, representative histograms of the distribution of HER2 expression for two TMA cores are shown in Figure 5D. By using these distributions, the data were replotted as an inverse cumulative distribution function to highlight the fraction of cells expressing greater than a given HER2 level (Figure 5E). This representation allows the visualization of the extreme heterogeneity of HER2 expression, with tumor cells expressing as few as approximately 40,000 HER2 per cell and up to >1,000,000 HER2 per cell, within the same sample. More important, plotted in this manner, our assay is able to quantitatively deliver two key measurements: HER2 numbers per cell and the percentage of cells expressing the indicated level of HER2.

Accordingly, the distribution of HER2 expression among all of the tumor cells in each of the TMA cores was analyzed through an inverse cumulative distribution function, as described in Figure 5, D and E, and the results are shown in Figure 5F. The plot for each individual core was color coded based on the traditional HER2 classification, shown in Figure 5C. The plots show that there is considerable variability of HER2 expression within any given sample. It is apparent that the HER2-negative patient samples have significantly fewer HER2 receptors per cell than the HER2positive samples.

\section{Clustering Analysis}

The HER2 quantitation, visualized using the inverse cumulative distribution function in Figure 5, indicated that there was substantial heterogeneity in the distribution of HER2 in the patient samples, particularly within the HER2-positive group. Because individual patient response to therapy within this group is variable, we next sought to explore whether there were distinct subgroups within the HER2positive group and how this compared with traditional testing methods. HER2 receptor distributions for each patient tumor core in the HER2-positive group were clustered using the K-means algorithm. This analysis identified three distinct subgroups in the traditional HER2-positive group, shown in Figure 6, A-C. The line colors in Figure 6, A-C, correspond to the IHC score for each sample. Visual inspection of these subgroups revealed three patterns: i) low and heterogeneous, samples that had heterogeneous expression dominated by comparatively lower HER 2 expression, but still classified as 
A

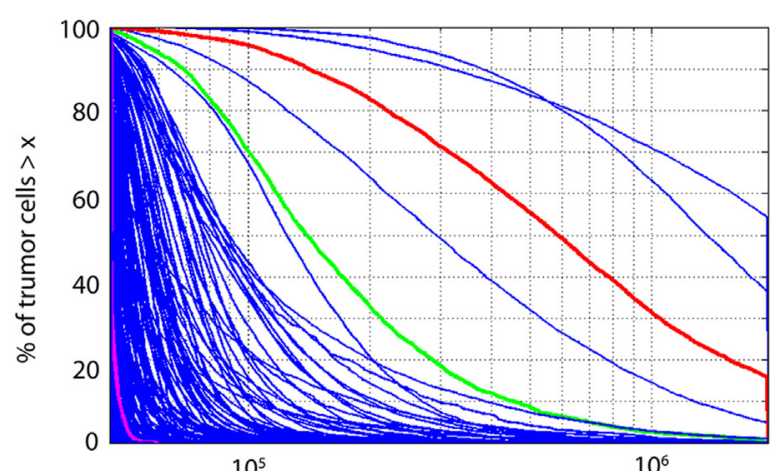

HER2 (No./cell)

C

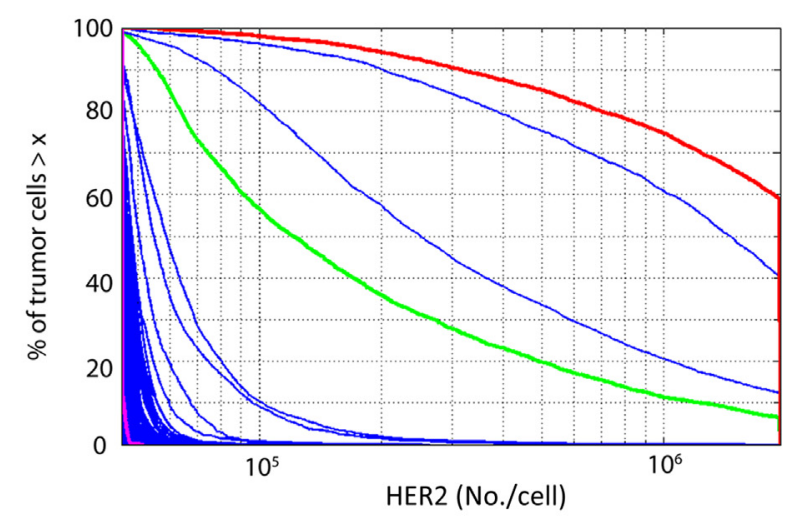

E

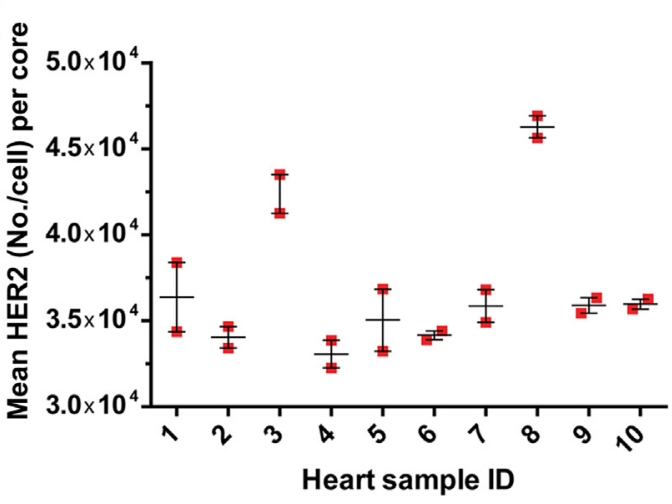

B
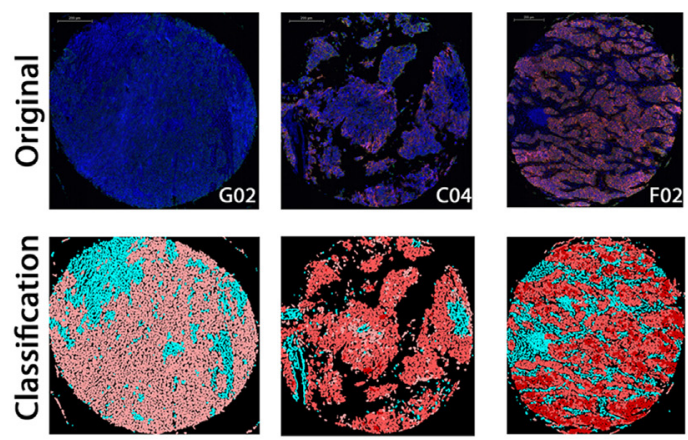

D
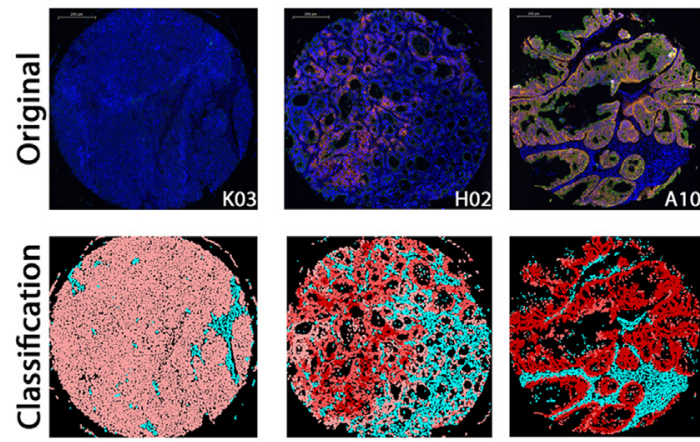

$\mathbf{F}$

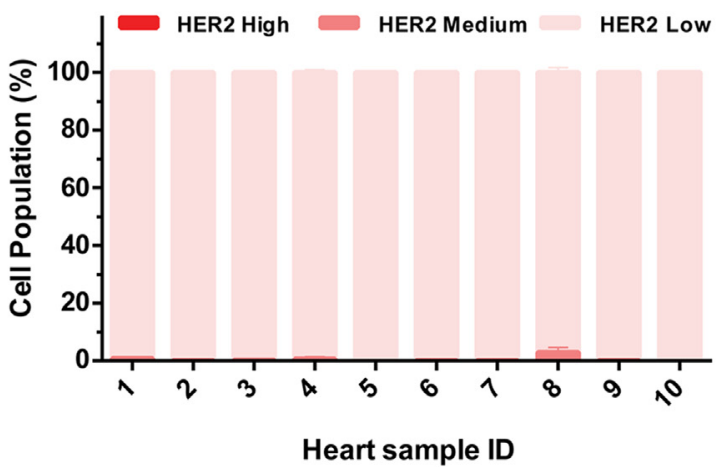

Figure 8 A: A bladder cancer TMA was stained as described in Materials and Methods in parallel with a standard cell pellet array. Slides were scanned with an Aperio ScanScope FL, and images were analyzed with Definiens Developer XD. The data for the individual TMA cores were plotted as an inverse cumulative distribution function. B: Three representative cores for different levels of HER2 expression, low (G02), medium (CO4), and high (F02), corresponding to the magenta, green, and red plots in A, respectively, are shown as original images (HER2, red; cytokeratin, green; DAPI, blue) or as classification views (HER2 high, red; HER2 medium, light red; HER2 low, pink; non-tumor cell/stroma, cyan). C: An ovarian cancer TMA was stained as described in Materials and Methods in parallel with a standard cell pellet array. Slides were scanned with an Aperio ScanScope FL and analyzed with Definiens Developer XD, and the data for the individual TMA cores were plotted as an inverse cumulative distribution function. D: Three representative cores for different levels of HER2 expression, low (K03), medium (H02), and high (A10), corresponding to the magenta, green, and red plots in C, respectively, are shown as original images (HER2, red; cytokeratin, green; DAPI, blue) or as classification views (HER2 high, red; HER2 medium, light red; HER2 low, pink; non-tumor cell/stroma, cyan). E: A heart TMA was stained with an anti-HER2 antibody and counterstained with DAPI. The slide was scanned with an Aperio ScanScope FL and analyzed with Definiens Developer XD. The mean HER2 per cell for the different TMA cores is plotted. F: The distribution of HER2 expression among the different populations: HER2 high ( $>$ approximately 1,000,000 HER2 per cell, red), HER2 medium (> approximately 150,000 and < approximately 1,000,000 HER2 per cell, light red), and HER2 low (< approximately 150,000 HER2 per cell, pink) is shown. Heart sample IDs correspond to the following diagnoses: 1 , chronic rheumatic valvular disease with calcification; 2, chronic rheumatic valvular disease; 3 , hepatocellular carcinoma embolus of cardiac atrium; 4, hypertrophic cardiomyopathy; 5, normal great arteries tissue; 6, normal cardiac atrium tissue; 7, normal myocardial tissue (focal mild hypertrophy); 8, normal auricle of heart tissue; 9, normal myocardial tissue (mild hypertrophy); and 10, normal myocardial tissue.

HER2 positive by traditional means (Figure 6A); ii) high and heterogeneous, samples exhibiting variable degrees of intermediate expression, but dominated by high HER2 expression (Figure 6B); and iii) unambiguously high, samples with most cells expressing high levels of HER2 (Figure 6C). All three subgroups show greater expression of HER2 than the traditional HER2-negative group (data not shown). In Figure 6, D and E, the proportion of each of our 
defined subgroups is shown within either the HercepTest $3+$ samples or the $2+/$ FISH-positive samples. The $3+$ patient samples were 59\% (13/22) unambiguously high, 18\% (4/22) high and heterogeneous, and the remaining 23\% (5/22) low and heterogeneous (Figure 6D). By contrast, the 2+/FISHpositive patients showed a broader distribution, with only $25 \%(5 / 20)$ unambiguously high, 60\% (12/20) high and heterogeneous, and the remaining 15\% (3/20) low and heterogeneous (Figure 6E).

Quantitative HER2 Measurement in Gastric and GEJ Tumors Intratumoral heterogeneity of HER 2 expression has been reported to be higher in gastric and GEJ cancers than in breast cancer. ${ }^{31,32}$ We used our assay to further characterize HER2 expression in 179 gastric and 57 GEJ whole tumor sections. Representative staining results for gastric and GEJ tumor sections are shown in Figure 7, A and B, respectively. Similar to the breast cancer results, the inverse cumulative distribution plots for the gastric (Figure 7C) and GEJ (Figure 7D) tumors show considerable variability of HER2 expression within any given sample and across samples. HER2 receptor distributions for each patient tumor clustered using the Kmeans algorithm. The clustering results revealed three subgroups of HER2 expression for GEJ and gastric samples. The three subgroups were as follows: i) unambiguously low, samples with nearly all cells expressing low levels of HER2 (data not shown); ii) low and heterogeneous, samples with a wide range of intermediate- to high-expressing cells (Figure 7E); and iii) high and heterogeneous, samples with a clear majority of cells expressing high levels of HER2 (Figure 7F). The high and heterogeneous gastric and GEJ samples were qualitatively distinct from the high and heterogeneous subclass found in the breast cancer samples. Only one sample was found in the gastric and GEJ samples that could be considered unambiguously high, where the HER 2 distribution is described by a single peak located beyond 1 million HER2 numbers per cell.

\section{Other Tumor and Tissue Types}

Because HER2 is expressed in a wide variety of cancers beyond breast and gastric, we sought to test the applicability of the assay in additional tumor types. To test the robustness of our assay and image analysis method, we also stained, classified, and scored tumors of bladder and ovarian origin. The inverse cumulative distribution functions for the analyzed bladder cancer TMA cores are shown in Figure 8A. Representative cores are shown in Figure $8 \mathrm{~B}$ as original images or as postimage analysis classification views. An ovarian cancer TMA was analyzed in a similar manner. The inverse cumulative distribution functions for the individual cores are shown in Figure 8C. Representative cores are shown in Figure 8D. The distinctly different morphological characteristics of the different tumors are adeptly handled by our analysis method.

Finally, we sought to demonstrate the applicability of our assay on normal tissue. We, therefore, chose to examine human heart tissue. In addition to its role in tumor progression, HER 2 has been shown to also have a protective role for cardiomyocytes exposed to stress. ${ }^{40,41} \mathrm{We}$ have previously shown that human stem cell-derived cardiomyocytes express low levels of HER 2 in vitro. ${ }^{42}$ A heart TMA, including both normal and diseased heart specimens, was stained for HER2 and counterstained with DAPI, in parallel with the previously described cell pellet array standard. The heart TMA and cell pellet array were analyzed as described in Materials and Methods, in a similar manner as the paired tumor TMAs and cell pellet arrays previously described. The mean HER2 number per cell for each of the heart cores analyzed is represented in Figure 8E. All of the cores showed mean HER2 numbers $<50,000$, including various types of diseased heart tissue. The distribution of the different HER2 cell populations (high/medium/low) is shown in Figure $8 \mathrm{~F}$. In all of the heart samples analyzed, $>95 \%$ of the cells in the core were classified as low HER2 expressing and showed mean HER2 numbers per cell in the same range of the lowest HER2-expressing cell line of the cell pellet array (ACHN, 45,000 HER2 per cell; Table 1).

\section{Discussion}

Herein, we detail the development and application of a quantitative immunofluorescence method for determining HER2 protein expression at the single-cell level in FFPE tissue samples. Two key aspects of this assay that define and differentiate it from previous work, such as AQUA, ${ }^{43,44}$ are as follows: the ability to quantitate HER2 staining at the single-cell level through the use of automated image analysis software that segments individual cells, and objective quantitation of HER2 in terms of molecules per cell based on a fully characterized standard curve.

We hypothesize that quantification at the single-cell level will be critical for assessing the impact of the heterogeneity of HER2 expression within a tumor on patient outcome, something that is not possible with current clinical HER2 assays. Furthermore, if used retrospectively, our assay could objectively determine the optimal degree of HER2 expression and the percentage of HER2-positive cells to use as a diagnostic cut point for prescribing HER2-directed therapies; this cut point is actively being debated in both breast and gastric cancers but cannot be determined satisfactorily using available assays. Patient response to trastuzumab in the clinic is variable, ranging from $36 \%$ to $79 \% .^{4}$ Further detailed analysis of the range of HER2 expression within biopsy specimens of patients treated with trastuzumab could help clarify the basis for such heterogeneous response rates. However, an appropriate control would be necessary to differentiate between prognostic and predictive effects of HER2 heterogeneity, and such patient samples are difficult to identify.

The use of automated image analysis software and a standard curve has the potential to minimize or possibly even remove reader subjectivity from the classification of 
HER2 status. There have been past attempts to incorporate automated image analysis in HER2 testing. ${ }^{45,46}$ This test would not replace a pathologist, but would be applied in conjunction with the pathologist who would verify that the tumor tissue was properly identified, stained, analyzed, and classified. The use of automated image analysis enables the evaluation of larger sections of tumor, and this will allow for a more accurate assessment of HER2 expression, ${ }^{47}$ which may result in improved patient stratification in the clinic and, possibly, improved therapeutic response rates.

Our analysis of breast cancer tissue samples indicated that FISH does an excellent job of classifying samples into those that have relatively high or low HER2 protein expression, and FISH agreed well with our classifying the $2+$ patients in terms of the mean protein expression levels. Overall, samples with HER2 gene amplification had an accompanying elevation in HER 2 protein levels, as measured by our assay. However, our clustering analysis indicated that there is substantial heterogeneity within both the HER2 high and low populations. Furthermore, HercepTest analysis of the FISH-positive samples did not capture the full extent of the heterogeneity. Our assay further demonstrates that the current testing paradigm, combining HercepTest and FISH, is an effective way to correctly classify the highest HER2expressing patients. However, our clustering of HER2 expression indicated that there are subtle, but reproducible, patterns of HER2 heterogeneity within traditional definitions of HER2 positivity. The clinical significance of these subgroups is not yet known and requires further investigation.

Our assessment of HER2 expression in gastric and GEJ whole tumor sections indicated that there is substantial heterogeneity in these tumor types as well. Our clustering analysis revealed three broad subgroups of HER2 expression in these tumors: unambiguously low, low and heterogeneous, and high and heterogeneous. The key distinction between the low and heterogeneous and high and heterogeneous subgroups was that the latter was characterized by more cells expressing intermediate to high levels of HER2. The patterns of heterogeneity in the gastric and GEJ samples shared some similarity, with heterogeneity found in breast cancer (compare low and heterogeneous in breast versus gastric). However, the gastric and GEJ samples also exhibited a qualitatively distinct pattern of heterogeneity (compare high and heterogeneous in gastric versus breast). A recent publication showed that heterogeneous HER2 amplification in patients with esophageal adenocarcinoma was associated with worse survival outcomes, suggesting that quantitative assessments of HER2 heterogeneity may be predictive of altered patient outcomes. ${ }^{48}$

The greatest opportunity for this assay may be in indications such as bladder, endometrial, or lung cancer, in which HER2 measurement has not yet been standardized. A correlation between HER2 amplification and disease stage was found in bladder cancer, in which approximately $14.2 \%$ of grade 3 tumors (versus $1.1 \%$ of grade 1 tumors) showed amplification. ${ }^{8}$ Bladder cancer is particularly interesting because it appears to be a cancer type in which overexpression of the HER2 protein is not always accompanied by gene amplification. ${ }^{6}$ Such cases might be particularly well suited for treatment with HER2-directed therapies, such as HER2-targeted liposomal doxorubicin ${ }^{42}$ or anti-HER2HER3-bispecific antibodies, ${ }^{49}$ that do not rely on addiction to HER2 signaling for their mechanism of action but only require elevated protein expression.

Our standard curve was characterized by high reproducibility between different cell array production batches and between staining runs performed by different investigators on different days. We did not find significant differences between HER2 antigen recognition for matched BT474-M3 tumor cells within the standard cell pellet array or within tumor xenografts (Supplemental Figure S1A), suggesting that the method of producing the cell array does not alter HER2 antigen recognition and that there are no significant differences in antigen recognition in the cell pellet array versus tissue. However, different biological characteristics of tumor cell growth in vivo versus in vitro could also result in different measured values, and this was observed in MDAMB-453 tumor xenografts (Supplemental Figure S1B).

This assay, however, is not without limitations. It is subject to the same challenges as most IHC assays regarding sample processing and fixation, and stability of protein antigens. ${ }^{16,30}$ Our results show that in tumors stored at $4^{\circ} \mathrm{C}$ (Supplemental Figure S2A), HER2 expression is preserved significantly better than in tumors stored at room temperature (Supplemental Figure S2B) before fixation (ischemia time). Especially for the longer ischemia times (24 and 96 hours or in the absence of fixation), HER2 expression was significantly affected in tumors stored at room temperature (Supplemental Figure S2C). Furthermore, the assay was also able to capture effects of different lengths of fixation on HER2 staining (Supplemental Figure S2D). These results are consistent with the published effects of sample processing and fixation on traditional IHC testing. More important, this suggests that the increased information content derived from our quantitative immunofluorescence method does not come at the expense of added sensitivity to preanalytical variables.

Because of the complexity of our assay, it is likely that it would necessitate implementation in a central laboratory. To demonstrate the clinical utility of this assay, it will be necessary to retrospectively analyze patient samples with measured clinical responses to an HER2-targeted therapy to set appropriate cut points; the clinical utility of the assay would then be assessed prospectively in a subsequent clinical trial. The objective and highly quantitative nature of our assay would be well positioned to determine whether there were more subtle relationships between response and HER2 expression and/or heterogeneity within samples that current clinically used assays cannot distinguish.

This work demonstrated the technical capabilities and potential utility of this assay technology platform using HER2 as an example. The HER2 field is a special case in 
which the clinical utility of its measurement has been demonstrated. The key opportunities for this assay may exist in the investigation of novel HER2-directed therapeutics and the study of HER2-expressing tumor types beyond breast and gastric. This assay can easily be extended to other HER family members, other cell surface targets, and intracellular proteins. More exploratory markers of patient response to therapy may ultimately prove to be the most clinically useful applications for this technology.

\section{Acknowledgments}

We thank Sam Agresta and Helen Lee for helpful discussions; Iga Sienczylo for technical assistance; Birgit Schoeberl, Ulrik Nielsen, Jonathan Fitzgerald, Frank Gibbons, and Bill Kubasek for taking the early and important steps toward developing quantitative IHC assays; Jinyan $\mathrm{Du}$ for the contribution to the development of the computational methods; and HistoRx (Branford, CT) for assistance with early development of the HER2 immunofluorescent staining method.

\section{Supplemental Data}

Supplemental material for this article can be found at http://dx.doi.org/10.1016/j.ajpath.2013.07.015.

\section{References}

1. Petrelli F, Barni S: Role of HER2-neu as a prognostic factor for survival and relapse in pT1a-bNOM0 breast cancer: a systematic review of the literature with a pooled-analysis. Med Oncol 2012, 29: $2586-2593$

2. Scholl S, Beuzeboc P, Pouillart P: Targeting HER2 in other tumor types. Ann Oncol 2001, 12(Suppl 1):S81-S87

3. Sauter G, Lee J, Bartlett JM, Slamon DJ, Press MF: Guidelines for human epidermal growth factor receptor 2 testing: biologic and methodologic considerations. J Clin Oncol 2009, 27:1323-1333

4. Bullock K, Blackwell K: Clinical efficacy of taxane-trastuzumab combination regimens for HER-2-positive metastatic breast cancer. Oncologist 2008, 13:515-525

5. Paik S, Kim C, Wolmark N: HER2 status and benefit from adjuvant trastuzumab in breast cancer. N Engl J Med 2008, 358: $1409-1411$

6. Simonetti S, Russo R, Ciancia G, Altieri V, De Rosa G, Insabato L: Role of polysomy 17 in transitional cell carcinoma of the bladder: immunohistochemical study of HER2/neu expression and fish analysis of c-erbB-2 gene and chromosome 17. Int J Surg Pathol 2009, 17: $198-205$

7. Caner V, Turk NS, Duzcan F, Tufan NL, Kelten EC, Zencir S, Dodurga Y, Bagci H, Duzcan SE: No strong association between HER2/neu protein overexpression and gene amplification in high-grade invasive urothelial carcinomas. Pathol Oncol Res 2008, 14:261-266

8. Simon R, Atefy R, Wagner U, Forster T, Fijan A, Bruderer J, Wilber K, Mihatsch MJ, Gasser T, Sauter G: HER-2 and TOP2A gene amplification in urinary bladder carcinoma [in German]. Verh Dtsch Ges Pathol 2002, 86:176-183

9. Fleming GF, Sill MW, Darcy KM, McMeekin DS, Thigpen JT, Adler LM, Berek JS, Chapman JA, DiSilvestro PA, Horowitz IR, Fiorica JV: Phase II trial of trastuzumab in women with advanced or recurrent HER2-positive endometrial carcinoma: a Gynecologic Oncology Group study. Gynecol Oncol 2010, 116:15-20

10. Santin AD, Bellone S, Van Stedum S, Bushen W, De Las Casas LE, Korourian S, Tian E, Roman JJ, Burnett A, Pecorelli S: Determination of HER2/neu status in uterine serous papillary carcinoma: comparative analysis of immunohistochemistry and fluorescence in situ hybridization. Gynecol Oncol 2005, 98:24-30

11. Konecny GE, Santos L, Winterhoff B, Hatmal M, Keeney GL, Mariani A, Jones M, Neuper C, Thomas B, Muderspach L, Riehle D, Wang HJ, Dowdy S, Podratz KC, Press MF: HER2 gene amplification and EGFR expression in a large cohort of surgically staged patients with nonendometrioid (type II) endometrial cancer. Br J Cancer 2009, 100:89-95

12. Kuyama S, Hotta K, Tabata M, Segawa Y, Fujiwara Y, Takigawa N, Kiura K, Ueoka H, Eguchi K, Tanimoto M: Impact of HER2 gene and protein status on the treatment outcome of cisplatin-based chemoradiotherapy for locally advanced non-small cell lung cancer. J Thorac Oncol 2008, 3:477-482

13. Pellegrini C, Falleni M, Marchetti A, Cassani B, Miozzo M, Buttitta F, Roncalli M, Coggi G, Bosari S: HER-2/Neu alterations in non-small cell lung cancer: a comprehensive evaluation by real time reverse transcription-PCR, fluorescence in situ hybridization, and immunohistochemistry. Clin Cancer Res 2003, 9:3645-3652

14. Yonesaka K, Zejnullahu K, Okamoto I, Satoh T, Cappuzzo F, Souglakos J, Ercan D, Rogers A, Roncalli M, Takeda M, Fujisaka Y, Philips J, Shimizu T, Maenishi O, Cho Y, Sun J, Destro A, Taira K, Takeda K, Okabe T, Swanson J, Itoh H, Takada M, Lifshits E, Okuno K, Engelman JA, Shivdasani RA, Nishio K, Fukuoka M, Varella-Garcia M, Nakagawa K, Janne PA: Activation of ERBB2 signaling causes resistance to the EGFR-directed therapeutic antibody cetuximab. Sci Trans1 Med 2011, 3:99ra86

15. Lammers RJ, Witjes JA: Discussion on the influence of HER2 status on the clinical outcome of bladder cancer continues. Expert Rev Anticancer Ther 2011, 11:853-858

16. Bai Y, Tolles J, Cheng H, Siddiqui S, Gopinath A, Pectasides E, Camp RL, Rimm DL, Molinaro AM: Quantitative assessment shows loss of antigenic epitopes as a function of pre-analytic variables. Lab Invest 2011, 91:1253-1261

17. Fritzsche FR, Kristiansen G, Boesl A, Burkhardt M, Pahl S, Dankof A, Dietel M, Dahl E: Tissue pretreatment with formic acid might lower HercepTest scores in breast cancer. Diagn Mol Pathol 2006, 15: $237-242$

18. Jacobs TW, Gown AM, Yaziji H, Barnes MJ, Schnitt SJ: Comparison of fluorescence in situ hybridization and immunohistochemistry for the evaluation of HER-2/neu in breast cancer. J Clin Oncol 1999, 17: 1974-1982

19. Siddiqui S, Rimm DL: Pre-analytic variables and phospho-specific antibodies: the Achilles heel of immunohistochemistry. Breast Cancer Res 2010, 12:113

20. Portier BP, Wang Z, Downs-Kelly E, Rowe JJ, Patil D, Lanigan C, Budd GT, Hicks DG, Rimm DL, Tubbs RR: Delay to formalin fixation "cold ischemia time": effect on ERBB2 detection by in-situ hybridization and immunohistochemistry. Mod Pathol 2013, 26: $1-9$

21. Press MF, Sauter G, Bernstein L, Villalobos IE, Mirlacher M, Zhou JY, Wardeh R, Li YT, Guzman R, Ma Y, Sullivan-Halley J, Santiago A, Park JM, Riva A, Slamon DJ: Diagnostic evaluation of HER-2 as a molecular target: an assessment of accuracy and reproducibility of laboratory testing in large, prospective, randomized clinical trials. Clin Cancer Res 2005, 11:6598-6607

22. Schnitt SJ, Jacobs TW: Current status of HER2 testing: caught between a rock and a hard place. Am J Clin Pathol 2001, 116:806-810

23. Rüschoff J, Hanna W, Bilous M, Hofmann M, Osamura RY, PenaultLlorca F, van de Vijver M, Viale G: HER2 testing in gastric cancer: a practical approach. Mod Pathol 2012, 25:637-650

24. Choritz H, Busche G, Kreipe H: Quality assessment of HER2 testing by monitoring of positivity rates. Virchows Arch 2011, 459:283-289 
25. Hicks DG, Tubbs RR: Assessment of the HER2 status in breast cancer by fluorescence in situ hybridization: a technical review with interpretive guidelines. Hum Pathol 2005, 36:250-261

26. Yeh IT, Martin MA, Robetorye RS, Bolla AR, McCaskill C, Shah RK, Gorre ME, Mohammed MS, Gunn SR: Clinical validation of an array CGH test for HER2 status in breast cancer reveals that polysomy 17 is a rare event. Mod Pathol 2009, 22:1169-1175

27. Tse CH, Hwang HC, Goldstein LC, Kandalaft PL, Wiley JC, Kussick SJ, Gown AM: Determining true HER2 gene status in breast cancers with polysomy by using alternative chromosome 17 reference genes: implications for anti-HER2 targeted therapy. J Clin Oncol 2011, 29:4168-4174

28. Wolff AC, Hammond ME, Schwartz JN, Hagerty KL, Allred DC, Cote RJ, Dowsett M, Fitzgibbons PL, Hanna WM, Langer A, McShane LM, Paik S, Pegram MD, Perez EA, Press MF, Rhodes A, Sturgeon C, Taube SE, Tubbs R, Vance GH, van de Vijver M, Wheeler TM, Hayes DF: American Society of Clinical Oncology/College of American Pathologists guideline recommendations for human epidermal growth factor receptor 2 testing in breast cancer. J Clin Oncol 2007, 25:118-145

29. Stenzinger A, von Winterfeld M, Aulmann S, Warth A, Weichert W, Denkert C, Ruschoff J, Dietel M, Klauschen F: Quantitative analysis of diagnostic guidelines for HER2-status assessment. J Mol Diagn 2012, 14:199-205

30. Moatamed NA, Nanjangud G, Pucci R, Lowe A, Shintaku IP, Shapourifar-Tehrani S, Rao N, Lu DY, Apple SK: Effect of ischemic time, fixation time, and fixative type on HER2/neu immunohistochemical and fluorescence in situ hybridization results in breast cancer. Am J Clin Pathol 2011, 136:754-761

31. Khoury T, Sait S, Hwang H, Chandrasekhar R, Wilding G, Tan D, Kulkarni S: Delay to formalin fixation effect on breast biomarkers. Mod Pathol 2009, 22:1457-1467

32. Minot DM, Kipp BR, Root RM, Meyer RG, Reynolds CA, Nassar A, Henry MR, Clayton AC: Automated cellular imaging system III for assessing HER2 status in breast cancer specimens: development of a standardized scoring method that correlates with FISH. Am J Clin Pathol 2009, 132:133-138

33. Turashvili G, Leung S, Turbin D, Montgomery K, Gilks B, West R, Carrier M, Huntsman D, Aparicio S: Inter-observer reproducibility of HER2 immunohistochemical assessment and concordance with fluorescent in situ hybridization (FISH): pathologist assessment compared to quantitative image analysis. BMC Cancer 2009, 9:165

34. Yang J, Luo H, Li Y, Li J, Cai Z, Su X, Dai D, Du W, Chen T, Chen M: Intratumoral heterogeneity determines discordant results of diagnostic tests for human epidermal growth factor receptor (HER) 2 in gastric cancer specimens. Cell Biochem Biophys 2012, 62:221-228

35. Lee S, de Boer WB, Fermoyle S, Platten M, Kumarasinghe MP: Human epidermal growth factor receptor 2 testing in gastric carcinoma: issues related to heterogeneity in biopsies and resections. Histopathology 2011, 59:832-840

36. McCabe A, Dolled-Filhart M, Camp RL, Rimm DL: Automated quantitative analysis (AQUA) of in situ protein expression, antibody concentration, and prognosis. J Natl Cancer Inst 2005, 97: $1808-1815$
37. Faratian D, Christiansen J, Gustavson M, Jones C, Scott C, Um I, Harrison DJ: Heterogeneity mapping of protein expression in tumors using quantitative immunofluorescence. J Vis Exp 2011, (56): e3334

38. Simpson EH: Measurement of diversity. Nature 1949, 163:688

39. Schoeberl B, Pace EA, Fitzgerald JB, Harms BD, Xu L, Nie L, Linggi B, Kalra A, Paragas V, Bukhalid R, Grantcharova V, Kohli N, West KA, Leszczyniecka M, Feldhaus MJ, Kudla AJ, Nielsen UB: Therapeutically targeting ErbB3: a key node in ligandinduced activation of the ErbB receptor-PI3K axis. Sci Signal 2009, 2:ra31

40. Feldman AM, Lorell BH, Reis SE: Trastuzumab in the treatment of metastatic breast cancer: anticancer therapy versus cardiotoxicity. Circulation 2000, 102:272-274

41. Gianni L, Salvatorelli E, Minotti G: Anthracycline cardiotoxicity in breast cancer patients: synergism with trastuzumab and taxanes. Cardiovasc Toxicol 2007, 7:67-71

42. Reynolds JG, Geretti E, Hendriks BS, Lee H, Leonard SC, Klinz SG, Noble CO, Lücker PB, Zandstra PW, Drummond DC, Olivier KJ Jr., Nielsen UB, Niyikiza C, Agresta SV, Wickham TJ: HER2-targeted liposomal doxorubicin displays enhanced anti-tumorigenic effects without associated cardiotoxicity. Toxicol Appl Pharmacol 2012, 262: $1-10$

43. Giltnane JM, Molinaro A, Cheng H, Robinson A, Turbin D, Gelmon K, Huntsman D, Rimm DL: Comparison of quantitative immunofluorescence with conventional methods for HER2/neu testing with respect to response to trastuzumab therapy in metastatic breast cancer. Arch Pathol Lab Med 2008, 132:1635-1647

44. Dimou A, Agarwal S, Anagnostou V, Viray H, Christensen S, Gould Rothberg B, Zolota V, Syrigos K, Rimm DL: Standardization of epidermal growth factor receptor (EGFR) measurement by quantitative immunofluorescence and impact on antibody-based mutation detection in non-small cell lung cancer. Am J Pathol 2011, 179:580-589

45. Wang S, Saboorian MH, Frenkel EP, Haley BB, Siddiqui MT, Gokaslan S, Wians FH Jr., Hynan L, Ashfaq R: Assessment of HER2/neu status in breast cancer: Automated Cellular Imaging System (ACIS)-assisted quantitation of immunohistochemical assay achieves high accuracy in comparison with fluorescence in situ hybridization assay as the standard. Am J Clin Pathol 2001, 116:495-503

46. Kilpatrick MW, Sheehan CE, Marganski WA, Tafas T, Ross MS, Ross JS: Determination of HER2 gene status by fully automated fluorescence microscopy. Anal Quant Cytol Histol 2011, 33:205-210

47. Tolles J, Bai Y, Baquero M, Harris LN, Rimm DL, Molinaro AM: Optimal tumor sampling for immunostaining of biomarkers in breast carcinoma. Breast Cancer Res 2011, 13:R51

48. Yoon HH, Shi Q, Sukov WR, Lewis MA, Sattler CA, Wiktor AE, Wu TT, Diasio RB, Jenkins RB, Sinicrope FA: Adverse prognostic impact of intratumor heterogeneous HER2 gene amplification in patients with esophageal adenocarcinoma. J Clin Oncol 2012, 30:3932-3938

49. McDonagh CF, Huhalov A, Harms BD, Adams S, Paragas V, Oyama S, Zhang B, Luus L, Overland R, Nguyen S, Gu J, Kohli N, Wallace M, Feldhaus MJ, Kudla AJ, Schoeberl B, Nielsen UB: Antitumor activity of a novel bispecific antibody that targets the ErbB2/ErbB3 oncogenic unit and inhibits heregulin-induced activation of ErbB3. Mol Cancer Ther 2012, 11:582-593 